\title{
Chromosome Y et stérilité masculine
}

\author{
PH. BERTA
}

Human Molecular Genetics group, Institut de génétique humaine, UPR1142 CNRS*

\begin{abstract}
RESUME
L'origine génétique d'une grande proportion des stérilités idiopathiques est aujourd'hui largement reconnue. Des corrélations génotype-phénotype réalisées depuis plusieurs années ont permis d'établir que dans sa partie spécifique du sexe, le chromosome $Y$ pourrait être le support génétique non exclusif d'un grand nombre de ces anomalies. Cette hypothèse se trouve aujourd'hui confortée par la meilleure définition moléculaire des intervalles de délétion de l'Y chez de tels patients. La mise en Iumière de différents gènes ou famille de gènes localisés dans ces loci du chromosome $Y$ conservés sur l'Y de différents mammifères mais aussi leurs profils d'expression au cours de la spermatogenèse permettent de postuler leur implication dans de telles stérilités. Cette mini-revue a pour but de dresser un état actuel de nos connaissances dans ce domaine.
\end{abstract}

\section{INTRODUCTION}

Si la stérilité affecte environ $15 \%$ des couples, dans un peu plus d'un tiers des cas, c'est le partenaire masculin qui est en cause, soit environ 1 homme sur 25. Les causes même de cette stérilité sont multiples, on peut citer pêle-mêle :

- obstruction des conduits spermatiques;

- agénésie des canaux déférents ;

- viscosité du sperme ;
- dysfonction éjaculatoire ;

- problèmes endocriniens (hypogonadisme hypogonadotrope);

- nécrospermie ;

- varicocèle ;

- défauts de spermatogenèse.

Ces anomalies peuvent donc être génétiques, mécaniques ou encore liées à des facteurs d'environnement. Quand la cause sera inconnue, on parlera de stérilité idiopathique. L'étiologie proposée reste le plus souvent de nature descriptive et l'on peut jouer sur la définition de "stérilité idiopathique" jusqu'à y regrouper environ $40 \%$ des stérilités masculines. Cette connaissance insuffisante de la pathogenèse des anomalies de la spermatogenèse justifie les limites actuelles dans les approches thérapeutiques.

Quelle que soit l'origine de cette stérilité, on aura donc recours à des techniques d'assistance médicale à la procréation et plus particulièrement aujourd'hui d dinjection intracytoplasmique de sperme (ICSI). La nécessité de tests génétiques et de conseil génétique pour les patients candidats à l'ICSI se pose aujourd'hui de façon aiguë $[2,5]$.

En effet, les défauts génétiques qui seraient éliminés ou perdus par des moyens naturels (absence de fécondation correcte) peuvent être transmis à la descendance.

* 141 rue de la Cardonille, 34396 Montpellier cedex 5, France Tél: 0499619955 - Fax : 0499619901 e-mail : berta@igh.cnrs.fr 
Des études réalisées au cours de ces 5 dernières années, démontrent que parmi les $10 \%$ d'hommes diagnostiqués comme oligo ou azoospermiques en absence de troubles mécaniques, environ $15 \%$ d'entre eux sont porteurs de délétions du seul bras long du chromosome $\mathrm{Y}$ et conduisant par la même à la perte de gènes vitaux pour la production de sperme.

C'est cette seule catégorie qui sera ici prise en considération au travers d'une revue de nos connaissances sur le chromosome $Y$ humain et des facteurs de fertilité qu'il encode $[6,14]$. Les gènes localisés sur le chromosome $\mathrm{Y}$ sont étudiés aujourd'hui facilement grâce à l'avancement de nos connaissances cartographiques mais aussi parce qu'il est par nature haploïde. Le chromosome Y n'est pas essentiel pour la vie, nombreuses sont celles qui peuvent en témoigner. Il est donc peu probable que des microdéletions de l'Y ne conduisent à autre chose que des problèmes de fertilité, ce qui limite les risques de l'ICSI.

\section{RESULTATS}

\section{Le chromosome Y humain}

Jusqu'en 1976, le chromosome Y n'était entrevu que sous la forme d'un "désert génétique", sauvé par la seule fonction "détermination du sexe" aujourd'hui égalée au gène SRY. Puis en 1976, les travaux de Tiepolo et Zuffardi et leurs premiers analyses de facteurs contrôlant la spermatogenèse rendaient nécessaire d'y supposer localisé un ou plusieurs facteurs nécessaires au bon déroulement de la spermatogenèse [17]. Enfin, très récemment, les travaux de David Page ont conduit à l'identification de plus d'une vingtaine de gènes ou de familles de gènes sur ce petit chromosome [10].

On peut schématiquement dissocier le chromosome $\mathrm{Y}$ en trois régions de tailles très inégales [3] (figure 1).

- Une région d'identité de séquence entre les chromosomes $\mathrm{Y}$ et $\mathrm{X}$ localisée à l'extrémité du bras court de l'Y ou région pseudo-autosomique. Cette dernière serait nécessaire à l'association des deux chromosomes sexuels au cours de la méiose, association accompagnée d'un chiasma unique et donc d'un échange de séquences ADN maintenant l'homologie de ces 2 régions, celle de l'X et celle de l'Y.

L'existence de ce chiasma permet la construction d'une carte méiotique de cette région puis le clonage de gènes pseudo-autosomiques en rien spécifiques de fonctions liées au sexe.

Pour exemple, on trouvera le gène codant le récepteur du facteur GM-CSF, une sous-unité du récepteur interleukine-3, une translocase $\mathrm{ADP} / \mathrm{ATP}$, PHOG un gène candidat pour justifier la petite taille des individus Turner ou encore une methyl-transferase.

Tous ces gènes, pour répondre à la mise en place du phénomène de compensation de dose par inactivation de l'un des chromosomes $\mathrm{X}$ femelles, échappent à cette inactivation assurant 2 doses chez l'homme comme chez la femme.

- La région spécifique du sexe (enfin le croyaiton) regroupe le reste de ce petit chromosome, à savoir la région déterminante du sexe, spécifique du sexe mâle accolée à la région pseudo-autosomique et qui a conduit au clonage au facteur déterminant SRY. Elle regroupe aussi la partie plus centromérique du bras court et toute la portion euchromatique du bras long. On y retrouve deux grandes catégories de gènes :

1. Des gènes non liés à des fonctions spécifiques du mâle et qui permettent en raison de la présence d'un homologue sur le $\mathrm{X}$ échappant à l'inactivation, le maintien d'un même nombre de doses.

\section{Exemples : ZFX/ZFY \\ AMELX/AMELY \\ MIC2 \\ SOX 3/SRY ?}

Ces gènes apparaissent exprimés avec un spectre assez large dans de nombreux tissus et ont donc tous une contrepartie sur le chromosome $\mathrm{X}$.

2. Et enfin des gènes exprimés spécifiquement ou de façon prédominante dans le testicule, gènes présents, comme nous le verrons, essentiellement en multiples copies. 


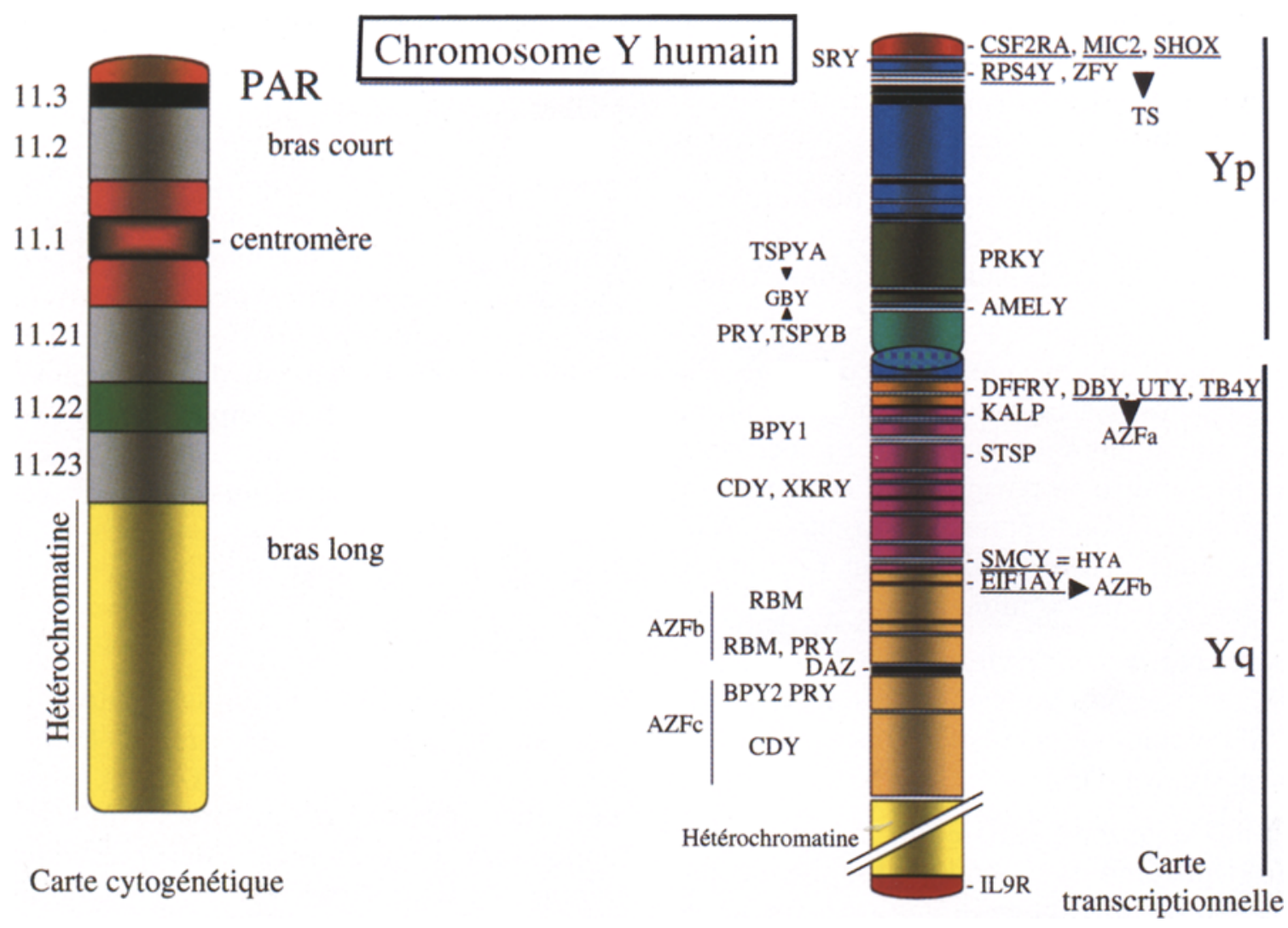

\section{Figure 1}

- Une grande région hétérochromatique de taille variable d'un chromosome $\mathrm{Y}$ à l'autre, d'un individu à l'autre.

\section{Mutations du chromosome Y et stérilités masculines.}

Les premières formes d'infertilité mâle idiopathiques mises en lumière par la cytogénétique sont des aneuploïdies chromosomiques avec caryotype 47,XXY d'une fréquence $1 / 500$ ou syndrome de Klinefelter. Au-delà, des altérations visibles en cytogénétique classique chez des patients azoospermes ou oligospermes sont décelées dans environ $10-20 \%$ des cas et justifient pleinement ce premier type d'analyse.

Cependant des microdélétions de moins de $2.10^{6}$ nucléotides échappent à cette analyse. L'analyse de telles microdélétions ont mis en lumière 3 loci génétiques liés à la spermatogenèse sur le bras long du chromosome $\mathrm{Y}$, en Yq11 plus précisément confirmant les observations déjà anciennes de Tiepolo et Zuffardi [17]. Cette observation conduit à l'hypothèse d'un facteur azoospermique ou AZF dans cette région. Cette hypothèse a ensuite été renforcée par de nombreuses études tant au niveau cytogénétique qu'au niveau moléculaire. Une autre hypothèse ne pouvait cependant pas être ignorée. Selon cette deuxième hypothèse, ces anomalies grossières du chromosome $\mathrm{Y}$ perturberaient l'appariement ou la ségrégation des chromosomes sexuels durant la méiose provoquant ainsi une azoospermie. L'exclusion de cette deuxième hypothèse n'a été rendue possible qu'avec l'observation chez des patients stériles de microdélétions, non visibles cytogénétiquement, par l'utilisation de sondes ADN; ces microdélétions n'impliquent pas de régions connues pour s'apparier et recombiner avec le chromosome $\mathrm{X}$ et semblent donc moins à même de perturber le comportement des chromosomes sexuels au cours de la méiose.

a. Le facteur $A Z F$ : aspects cliniques

L'histologie génitale et testiculaire des hommes souffrants d'anomalies cytogénétiques en Yq11 ont rapidement suggéré une 
fonction complexe pour AZF. Ainsi sont observés des syndromes à "Sertoli cell only", l'absence de cellules post-méiotiques, des azoospermies ou des oligospermies.

Cette hétérogénéité suggère l'existence de trois intervalles délétionnels non chevauchants et donc plusieurs gènes dans cette région du chromosome $\mathrm{Y}$ contrôlant la spermatogenèse (figure 2).

L'établissement d'une carte de délétion de cette région grâce à la collection de STS de la région et parallèlement l'analyse moléculaire d'un grand nombre de patients ont conduit à la définition de 3 sous-régions distinctes, trois loci, puis trois facteurs $\mathrm{AZFa}$, b et c rendant compte des stérilités étudiées [19].

Chacun de ces loci intervient à un stade différent de la spermatogenèse et les caractéristiques histologiques correspondantes sont les suivantes (figure 3 ) :

AZFa : des délétions dans ce locus sont associées au syndrome de Sertoli Cell Only, absence totale de cellules germinales et à un petit volume testiculaire suggérant une anomalie prépubère au moment de la production des spermatogonies souches ou au cours de leur différenciation en spermatocytes.

AZFb : si les cellules germinales pré-méiotiques sont présentes en quantité normale dans les stérilités associés à des délétions de cette région, aucune cellule post-méiotique n'est observée. Ceci suggère une interruption à la puberté pendant ou avant l'étape méiotique.

Pour AFAa et AZFb, aucune cellule germinale mature n'est observée dans aucun des tubes séminifères et il ne semble pas y avoir de variabilité phénotypique de l'histologie des testicules des patients.

AZFc : L'histologie testiculaire des hommes délétés dans cette région est des plus diverses. On peut ainsi retrouver un phénotype proche du "Sertoli cell only", d'autres avec des cellules germinales à différentes étapes du processus spermatogénétique ou des patients avec des tubes vides côtoyant des tubes avec des spermatogonies et spermatocytes. On observe donc des cas d'azoospermies mais aussi des oligospermies.
Il existe aussi de rares cas, où un homme porteur de délétions du locus AZFc a pu avoir un fils, qui lui-même porteur de la même délétion s'est retrouvé azoospermique. Il reste difficile de réconcilier cette diversité phénotypique associée à une même délétion. Certaines hypothèses ont été avancées sur une origine commune de réduction du nombre de spermatozoïdes qui serait l'effet primaire suivi d'une dégénérescence dépendante de l'âge. On peut également relier cette variabilité phénotypique à la taille de la délétion impliquant un nombre différent de gènes ou à une mosaïque germinale. Enfin, ces observations peuvent résulter d'une pénétrance variable de ces délétions fonction du contexte génétique de l'individu.

\section{b. Le facteur $A Z F$ - analyse moléculaire}

Les cas d'infertilité cliniques peuvent donc aujourd'hui être facilement analysés par une large collection de primers PCR qui foisonnent dans la littérature et qui ont permis le développement de systèmes PCR multiplex qui comprennent ces régions du chromosome $\mathrm{Y}$ (figure 4).

On obtient ainsi un diagnostic rapide grâce à cette technique qu'il faudra ensuite confirmer pour être des délétions de novo absentes chez le père lui-même fertile.

Ces tests diagnostiques appellent plusieurs commentaires :

- Il semble important d'élargir cette analyse moléculaire en dehors de ces seuls loci AZF. On ne peut exclure à ce jour l'existence d'autres gènes de fertilité.

- Nous n'avons pas encore accès à une collection de marqueurs suffisamment étendue pour couvrir l'ensemble de Yq11 soit pratiquement 10 millions de nucléotides.

c. Des gènes candidats pour les fonctions $A Z F a$, $A Z F b$ et $A Z F c$

* Les gènes de la famille RBM pour RNA binding motif

Clonés à partir des régions délétées en AZFc, la famille des gènes RBM (anciennement YRRM) encodent des protéines de liaison à l'ARN [4, 7, 9]. Cependant, il s'agit d'une large famille de gènes et de pseudogènes également 


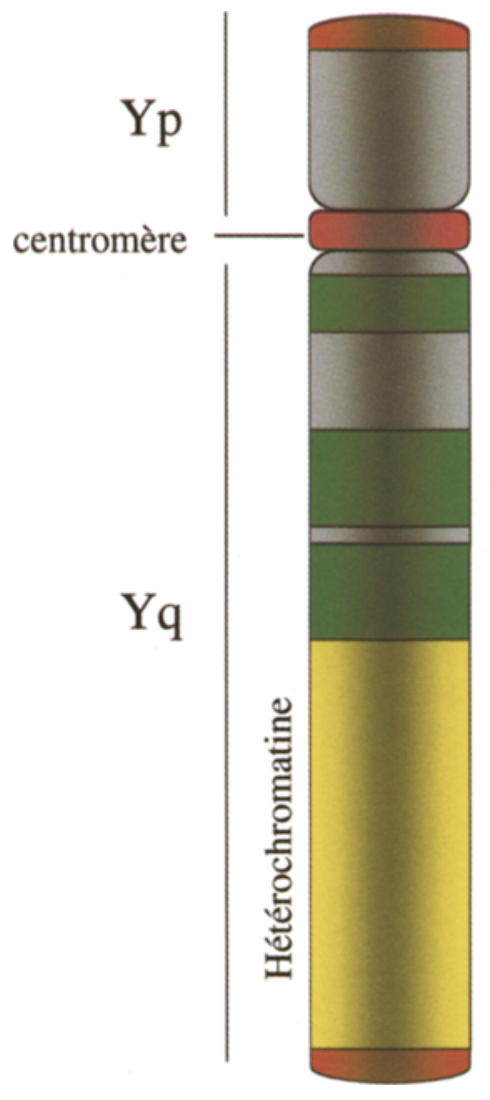

PAR

$\mathrm{AZFa} \longrightarrow$ Arrêt précoce (SCO syndrome)

$\mathrm{AZFb} \longrightarrow$ Arrêt préméiotique ou méiotique

$\mathrm{AZFc} \longrightarrow$ Arrêt postméiotique (oligospermie-azoospermie)

\section{Histologie testiculaire d'une azoospermie}
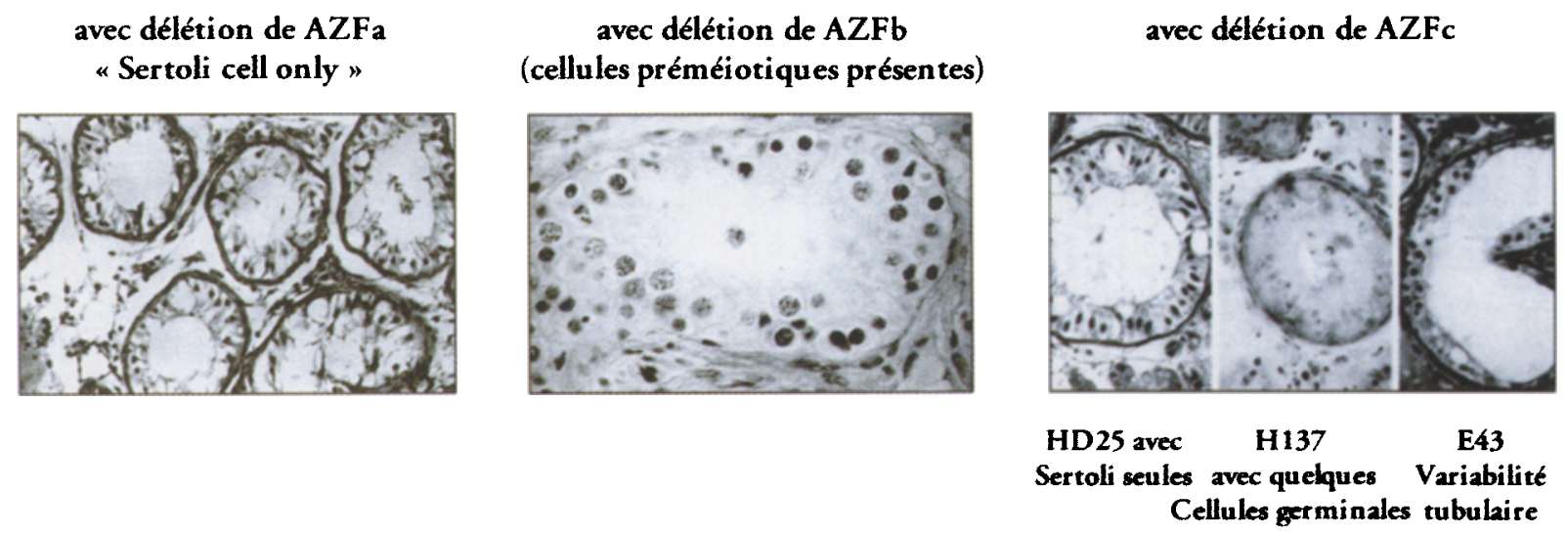

(d'apres Vogt, 1997)

Figure 3 


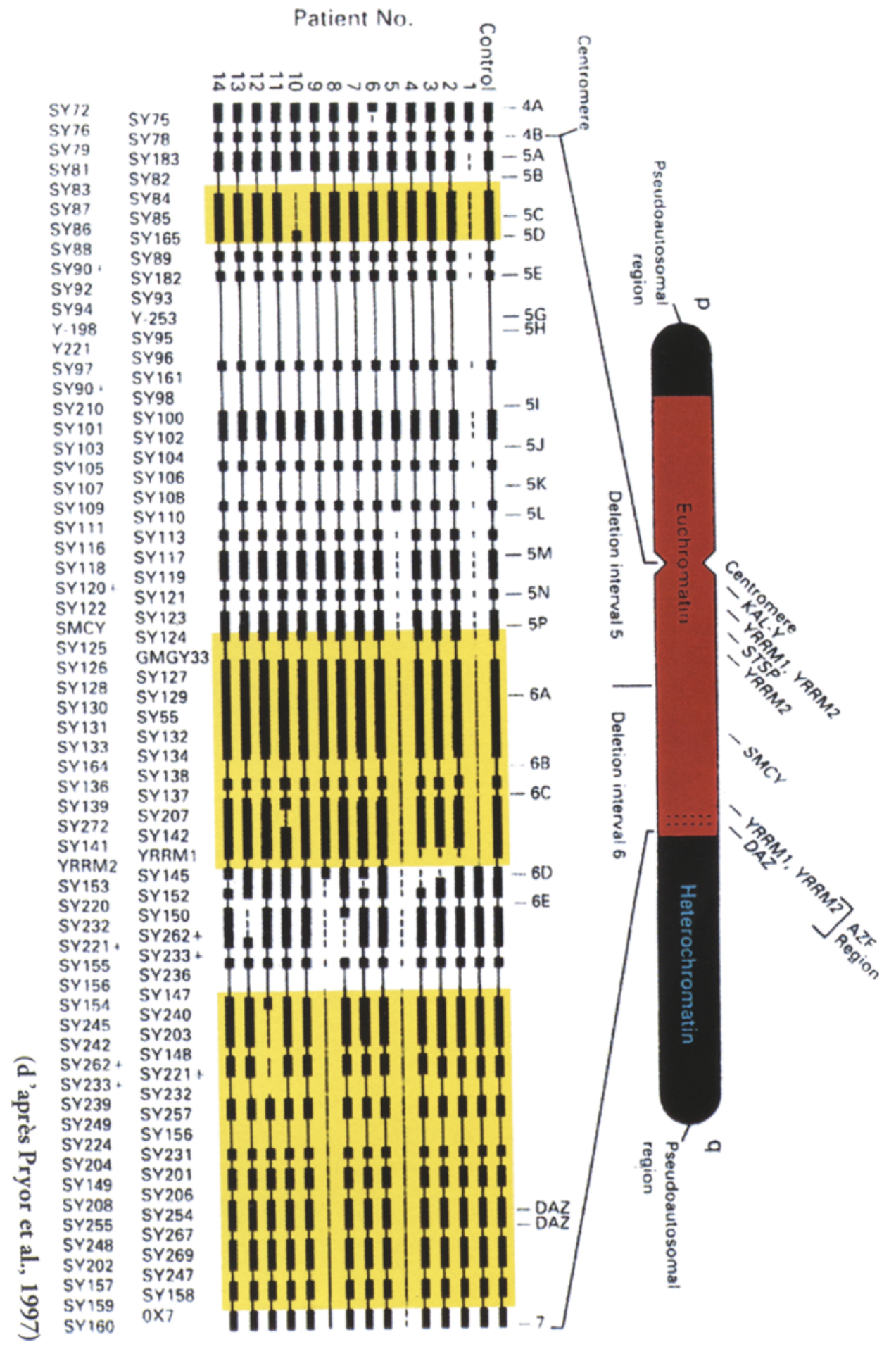

Figure 4 
localisés en AZFa et b mais aussi sur le bras court, d'où une estimation totale de plus de 20 gènes (figure 5).

D'expression restreinte à la lignée germinale mâle chez l'homme comme chez la souris, cette protéine est nucléaire et son expression restreinte aux différents stades de la spermatogenèse. De plus des travaux récents d'Howard Cooke montre que seuls les gènes RBM de la région $\mathrm{AZFb}$ semblent contribuer à cette expression. Il est intéressant de noter que seule la séquence RBM1 est conservée chez les mammifères métathériens. Il tirerait son origine d'une duplication ancestrale du gène hnRNPG sur l'Y suggérant sa contribution au cours du processus spermatogénétique au processing des pré-messagers. La nature multiple du gène RBM rend encore aujourd'hui très difficile sa caractérisation en tant que responsable de stérilités masculines.

Des délétions d'un ou plusieurs de ces gènes ont été observées dans certaines stérilités. Ce rôle au cours de la spermatogenèse est conforté par une expression restreinte au testicule. Le rôle exact en demeure inconnu mais son homologie avec le gène autosomique hnRNPG suggère un rôle dans le processing des ARN pré-messagers et dans leur transport.

\section{* Le gène $D A Z$}

Plus récemment, un autre gène fut cloné à partir de la région $\mathrm{AZFc}$. Ce gène fut dénommé DAZ pour "deleted in azoospermia" [13]. C'est un gène particulier parmi les gènes non spécifiques du chromosome $Y$, présent en plusieurs copies avec un homologue non pas sur le $\mathrm{X}$ mais sur un autosome [15, 16]. Là encore il s'agit d'un gène codant pour une protéine de liaison à l'ARN. De plus, DAZ est également membre d'une famille multigénique dont le gène SPGY ou un homologue autosomique DAZLA sur le chromosome 3 humain. Les gènes du chromosome 3 comme du chromosome Y sont exprimés dans le testicule. De plus des mutations du gène boule, homologue de DAZ chez la drosophile conduit à un arrêt méiotique et à la stérilité [8].

* Le gène TSPY

Présent comme RBM en 20 à 40 copies disper- sées le long du chromosome, il présente $30 \%$ d'identité protéique avec SET chez l'homme, le rat et la drosophile et code pour un facteur activateur du cycle cellulaire [1]. D'expression limitée aux spermatogonies, TSPY peut être impliqué dans la prolifération mitotique des cellules germinales. Son absence du chromosome $\mathrm{Y}$ de certains rongeurs soulève de nouvelles questions [11].

\section{* Futurs candidats.}

En 1997, l'identification dans la région spécifique du chromosome Y par l'équipe de David Page de 12 nouveaux gènes ou familles de gènes fournit de nouveaux candidats potentiels. Ainsi, si 7 d'entre eux sont exprimés dans le testicule et sont en multiple copies, 3 pourraient même être susceptibles d'interaction avec les acides nucléiques [10].

\section{DISCUSSION}

En considérant qu'il y a encore peu de temps aucune base moléculaire ne venait justifier les si nombreuses oligo et azoospermies, les études faites sur le chromosome $\mathrm{Y}$ et les approches PCR fournissent une possibilité diagnostique pour des stérilités d'origine non obstructive. La rapidité des acquis génétiques devrait permettre rapidement de compléter le catalogue de l'ensemble des gènes de fertilité localisés sur l'Y et d'entrevoir une meilleure corrélation phénotype - génotype. Cette corrélation reste encore difficile et la présence de familles multigéniques ne simplifie en rien la tâche.

Les implications de ces découvertes sont encore limitées :

- Une détection des délétions du chromosome $Y$ fournit un outil diagnostique pour l'infertilité et cela fournit souvent une explication importante pour le patient.

- On peut espérer la mise en place de traitement, mais dès aujourd'hui cela permet au clinicien de diriger le patient vers la reproduction assistée ou encore l'adoption.

L'utilisation de l'ICSI pourra se faire en toute conscience avec la nécessité d'expliquer plus tard à la descendance les risques de stérilité possibles, permettant une préparation au conseil et à la planification. 


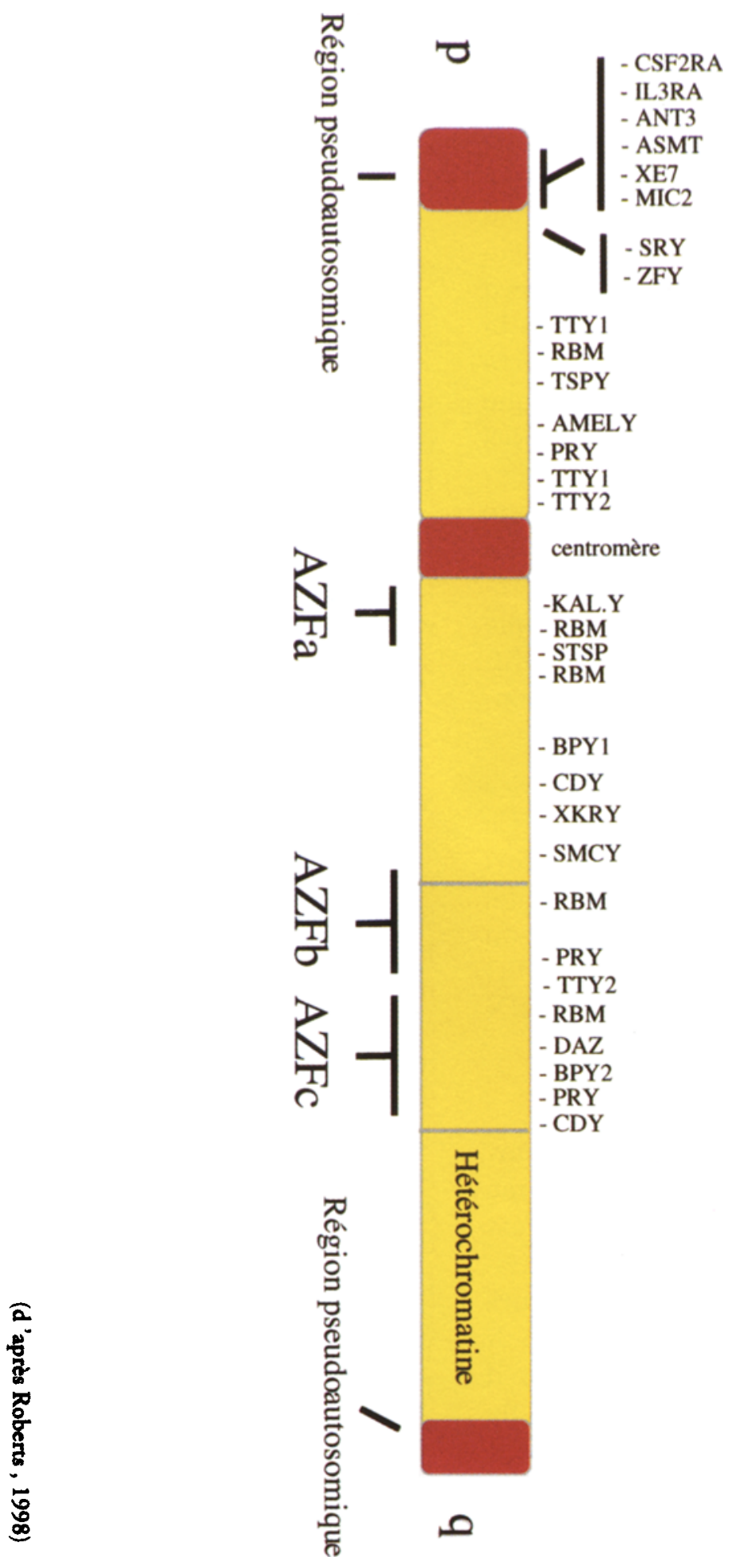

Figure 5 


\section{REFERENCES}

1. ARNEMANN J., JAKUBICZKA, S., THÜRING, S. et al. : Cloning and sequence analysis of a human Ychromosome-derived, testicular cDNA, TSPY. Genomics, 1991, 11: 108-114.

2. BOURGERON, T., BARBAUX, S., MCELREAVEY, K. et al. : La génétique de la stérilité masculine. Médecine/sciences, 1996, 12: I-VIII.

3. BURGOYNE, P.S. : The mammalian Y chromosome: a new perspective. Bioessays, 1998, 20: 363-366.

4. CHAI, N.N., SALIDO, E.C. AND YEN, P.H. : Multiple functional copies of the $R B M$ gene family, a spermatogenesis candidate on the human $\mathrm{Y}$ chromosome. Genomics, 1997, 45: 355-361.

5. CHANDLEY, A.C. : Chromosome anomalies and Y chromosome microdeletions as causal factors in male infertility. Hum. Reprod., 1998, 13 Suppl. 1: 45-50.

6. DE KRETSER, D.M. AND BURGER, H.G. : The Y chromosome and spermatogenesis. N. J. Engl. Med., 1997, 336, 576-578.

7. DELBRIDGE, M.L., HARRY, J.L., TODER, R. et al. : A human candidate spermatogenesis gene, $R B M 1$, is conserved and amplified on the marsupial $\mathrm{Y}$ chromosome. Nature Genet., 1997, 15: 131-136.

8. EBERHART, C.G., MAINES, J.Z. AND WASSERMAN, S.A. : Meiotic cell cycle requirement for a fly homologue of human Deleted in Azoospermia. Nature, 1996, 381:783-785.

9. ELLIOT, D.J., MILLAR, M.R., OGHENE, K. et al. : Expression of RBM in the nuclei of human germ cells is dependent on a critical region of the $\mathrm{Y}$ chromosome long arm. Proc.Natl.Acad.Sci. USA, 1997, 94: 3848 3853 .

10. LAHN BT, PAGE DC : Functional coherence of the human Y chromosome. Science, 1997, 24;278:675-80.

11. MAZEYRAT, S. MITCHELL, M.J. : Rodent Y chromosome TSPY gene is functional in rat and non-functional in mouse. Hum. Mol. Genet., 1998, 7: 557-562.

12. PRYOR, J.L., KENT-FIRST, M., MUALLEM, A. et al. : Microdeletions in the $\mathrm{Y}$ chromosome of infertile men. N. Engl. J. Med., 1997, 336: 534-539.

13. REIJO, R., LEE, T.Y., SALO, P. et al. : Diverse spermatogenic defects in humans caused by $\mathrm{Y}$ chromosome deletions encompassing a novel RNA-binding protein gene. Nature Genet. , 1995, 10: 383-393.

14. ROBERTS, K.P. : Y-chromosome deletions and male infertility: state of the art and clinical implications. J. Androl., 1998, 19: 255-259.

15. RUGGIU, M., SPEED, R., TAGGART, M. et al. : The mouse Dazla gene encodes a cytoplasmic protein essential for gametogenesis. Nature, 1997, 389: 73-77.

16. SAXENA, R., BROWN, L.G., HAWKINS, T. et al. : The $D A Z$ gene cluster on the human $\mathrm{Y}$ chromosome arose from an autosomal gene that was transposed, repeatedly amplified and pruned. Nature Genet., 1996, 14: 292-299.

17. TIEPOLO L. AND ZUFFARDI O. : Localization of factors controlling spermatogenesis in the nonfluorescent portion of the human $\mathrm{Y}$ chromosome long arm. Hum. Genet., 1976, 34, 119-124.

18. VOGT, P.H. : Genetics of idiopathic male infertility: Y chromosomal azzospermia factors ( $A F A a, A Z F b$, $A Z F c$ ). Baillieres Clin. Obstet. Gynaecol., 1997, 11: 773-795.

19. VOGT, P.H., EDELMANN, A., KIRSCH, S. et al. : Human $\mathrm{Y}$ chromosome azzospermia factors (AZF) mapped to different subregions in Yq11. Hum. Mol. Genet., 1996, 5: 933-943.

\author{
ABSTRACT \\ Chromosome $\mathbf{Y}$ et stérilité masculine \\ PH BERTA
}

Over the past decade the tools of cytogenetic investigations, the access to new molecular probes (sequence-tagged sites) and the refinement of chromosome maps have provided the possibility to gain insights on the involvment of the $Y$ chromosome as a support for part of the idiopathic male infertility. The first analysis of genotype-phenotype correlations established the existence of at least three separate intervals (known as Azoospermic Factor (AZF) loci) scattered through the sex specific sequences of the $Y$ chromosome. Diverse genes or families of genes have been cloned so far and now form candidate genes to be equated to one of these azoospermic factors. Our current knowledge is depicted in the following mini-review.

Key words: Ychromosome, Sterility, Genes 